\title{
Measurement of the magnetic field-dependent refractive index of magnetic fluids in bulk
}

\author{
Ting Liu (刘 婷 $)^{1}$, Xianfeng Chen (陈险峰 $)^{1,2}$, Ziyun Di (狄子昫) $)^{1,2}$, \\ Junfeng Zhang (张军锋 $)^{1,2}$, Xinwan Li (李新碗 $)^{1}$, and Jianping Chen (陈建平 $)^{1}$ \\ ${ }^{1}$ The State Key Laboratory on Fiber Optic Local Area Communication Networks and \\ Advanced Optical Communication Systems, Shanghai Jiao Tong University, Shanghai 200240 \\ ${ }^{2}$ Department of Physics, Shanghai Jiao Tong University, Shanghai 200240
}

Received August 14, 2007

\begin{abstract}
An optical alignment-free and highly accurate method is employed to measure the magnetic field-dependent refractive index of magnetic fluid (MF) in bulk. The measured refractive index decreases significantly with the increasing magnetic strength and then tends to saturate in the high intensity range. By applying a tunable magnetic field ranging between 0 and 1661 Oe, the maximum shift of the refractive index of MF in bulk is found to be 0.0231 .
\end{abstract}

OCIS codes: 290.3030, 160.3820, 060.2370.

Magnetic fluid (MF) is a kind of stable colloidal dispersion of ferromagnetic nano-particles in a suitable liquid carrier $^{[1]}$. Because the behaviors of ferromagnetic particles in MF are dependent on the external magnetic field, MF exhibits remarkable magneto-optical properties, such as birefringence $\mathrm{e}^{[2-7]}$, magnetochromatics ${ }^{[8,9]}$, and optical transmittance ${ }^{[10-13]}$. The refractive index of MF films is also shown to be magnetic field-dependent ${ }^{[14-16]}$. The property of magnetic field-dependent refractive index of MF has promising applications in fabricating optical switches ${ }^{[15,17]}$, tunable filters ${ }^{[18]}$, magnetic-field sensors, and other optical devices ${ }^{[15]}$.

Up to date, two methods, both employing reflection technique, have been reported to measure the refractive index of magnetic fluids. In 2002, Yang et al. successfully measured the magnetic field-dependent refractive index of MF films by total reflection technique ${ }^{[14]}$. However, the method requires sophisticated instrumentation, elaborate optical alignment and complicated data processing ${ }^{[19]}$. In 2005, Pu et al. developed an optical alignment-free and highly accurate method to measure the concentration and temperature-dependent refractive indices of MF in bulk ${ }^{[19]}$. In this paper, the method proposed by $\mathrm{Pu}$ et al. is employed to measure the refractive indices of MF in bulk under the changing external magnetic field.

Figure 1 shows the schematic diagram of the experimental setup to measure the magnetic field-dependent refractive index of MF in bulk.

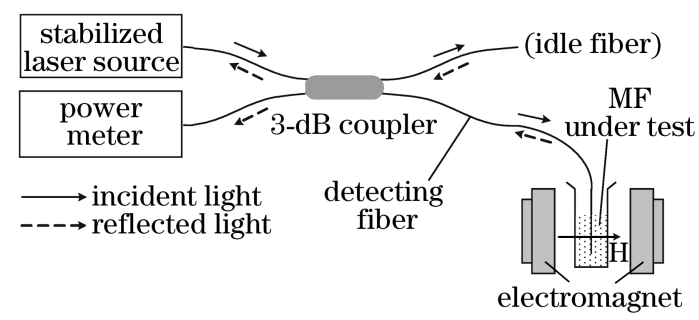

Fig. 1. Schematic diagram of experimental setup for measuring the field-dependent refractive index of MF in bulk.
The magnetic fluids used here is water-based $\mathrm{Fe}_{3} \mathrm{O}_{4}$ with the density of $1.2 \mathrm{~g} / \mathrm{mL}$. The incident light source is generated by a stabilized laser source which operates at the wavelength of $1550 \mathrm{~nm}$ and the room temperature is $20^{\circ} \mathrm{C}$. A 3-dB " $\mathrm{X}$ "-type single-mode fiber coupler is used to guide the incident light to the interface between the fiber core and MF. The magnetic fields of variable intensity are applied on MF by an electromagnet. The refractive indices of air, water and the fiber core are $n_{\text {air }}=1.0003, n_{\text {water }}=1.3330, n_{\mathrm{fc}}=1.46$, respectively.

The detecting tip of the fiber end with a flat cleaved face is immersed into air, water and MF under magnetic fields with different intensities and the power of the reflected light is measured by the power meter under each condition. The intrinsic reflection power $P_{0}$ of the experimental system can be obtained by solving the following equation ${ }^{[19]}$

$$
P_{0}=\frac{P_{\text {water }}\left(\frac{n_{\mathrm{fc}}-n_{\text {air }}}{n_{\mathrm{fc}}+n_{\text {air }}}\right)^{2}-P_{\text {air }}\left(\frac{n_{\mathrm{fc}}-n_{\text {water }}}{n_{\mathrm{fc}}+n_{\text {water }}}\right)^{2}}{\left(\frac{n_{\mathrm{fc}}-n_{\text {air }}}{n_{\mathrm{fc}}+n_{\text {air }}}\right)^{2}-\left(\frac{n_{\mathrm{fc}}-n_{\text {water }}}{n_{\mathrm{fc}}+n_{\text {water }}}\right)^{2}},
$$

where $P_{\text {air }}$ and $P_{\text {water }}$ are the measured power of the reflected light when the detecting tip is immersed into air and water, respectively.

The refractive index of MF can be calculated from the relation below ${ }^{[19]}$

$$
\begin{aligned}
& n_{\mathrm{mf}}=\frac{\left[1+\left(\frac{P_{\mathrm{mf}}-P_{0}}{P_{\mathrm{air}}-P_{0}}\right)\left(\frac{n_{\mathrm{fc}}-n_{\mathrm{air}}}{n_{\mathrm{fc}}+n_{\mathrm{air}}}\right)^{2}\right] n_{\mathrm{fc}}}{\left[1-\left(\frac{P_{\mathrm{mf}}-P_{0}}{P_{\mathrm{air}}-P_{0}}\right)\left(\frac{n_{\mathrm{fc}}-n_{\mathrm{air}}}{n_{\mathrm{fc}}+n_{\mathrm{air}}}\right)^{2}\right]} \\
& \pm \sqrt{\left\{\frac{\left[1+\left(\frac{P_{\mathrm{mf}}-P_{0}}{P_{\mathrm{air}}-P_{0}}\right)\left(\frac{n_{\mathrm{fc}}-n_{\mathrm{air}}}{n_{\mathrm{fc}}+n_{\mathrm{air}}}\right)^{2}\right] n_{\mathrm{fc}}}{\left[1-\left(\frac{P_{\mathrm{mf}}-P_{0}}{P_{\mathrm{air}}-P_{0}}\right)\left(\frac{n_{\mathrm{fc}}-n_{\mathrm{air}}}{n_{\mathrm{fc}}+n_{\mathrm{air}}}\right)^{2}\right]}-n_{\mathrm{fc}}^{2}-k_{\mathrm{mf}}^{2}\right.},
\end{aligned}
$$


where $P_{\mathrm{mf}}$ is the measured power of the reflected light when the detecting tip is immersed into MF. $k_{\mathrm{mf}}$ is the extinction coefficient of MF and can be gotten by the relation $k_{\mathrm{mf}}=\frac{\alpha_{\mathrm{mf}} \lambda}{4 \pi}$ (the absorption coefficient $\alpha_{\mathrm{mf}}$ of MF equals to $120 \mathrm{~cm}^{-1}$ and the wavelength is $1550 \mathrm{~nm}$ in our experiment). Given one set of measured data, two values of refractive indices of MF can be calculated from Eq. (2).

$$
\text { Because } \frac{\left[1+\left(\frac{P_{\mathrm{mf}}-P_{0}}{P_{\mathrm{air}}-P_{0}}\right)\left(\frac{n_{\mathrm{fc}}-n_{\mathrm{air}}}{n_{\mathrm{fc}}+n_{\mathrm{air}}}\right)^{2}\right]}{\left[1-\left(\frac{P_{\mathrm{mf}}-P_{0}}{P_{\mathrm{air}}-P_{0}}\right)\left(\frac{n_{\mathrm{fc}}-n_{\text {air }}}{n_{\mathrm{fc}}+n_{\text {air }}}\right)^{2}\right]}>1 \text {, the first part of }
$$

Eq. (2) is larger than $n_{\mathrm{fc}}$. The second part of Eq. (2) is constantly positive. The refractive index of the water based MF sample used in our experiment is smaller than $n_{\mathrm{fc}}$, so "-" should be selected in the calculation.

Using the experimental data to get $P_{0}$ first from Eq. (1) and then substituting $P_{0}$ into Eq. (2), the field-dependent refractive indices of $\mathrm{MF}$ can be calculated as shown in Fig. 2, the inset of which draws the experimental data of $P_{\mathrm{mf}}$ under the changing external magnetic field.

From Fig. 2 we can see that the refractive index of MF in bulk decreases significantly while the magnetic intensity increases from 0 to 1400 Oe and tends to saturate in the high intensity range. Under our experimental conditions, the magnetic field ranges between 0 and 1661 Oe, and the refractive index of MF in bulk decreases from 1.4475 to 1.4244 .

Our experiments show that the refractive index of MF in bulk decreases while the magnetic intensity increases, which seems in contradiction with the results of the study by Yang et al. ${ }^{[14-16]}$. By employing total reflection method, Yang et al. measured the field-dependent refractive indices of MF films with different thicknesses. Their results show that for MF films with various thicknesses, the field-dependent refractive indices are different. However, the trend that the refractive index of MF films increases with the increasing magnetic intensity is similar for MF films with different thicknesses ${ }^{[15]}$. MF in bulk can be considered as MF films with extremely high thickness. Thus, although what we explore is the property of MF in bulk rather than that of MF in films, the difference of thicknesses between them may not convincibly explain the contradiction.

We attribute the contradiction to the magneto-electric directive effect of ferrofluid ${ }^{[20]}$. The electric susceptibility $\chi$ of ferrofluids is dependent on the magnitude of the magnetic field and on the relative direction between the

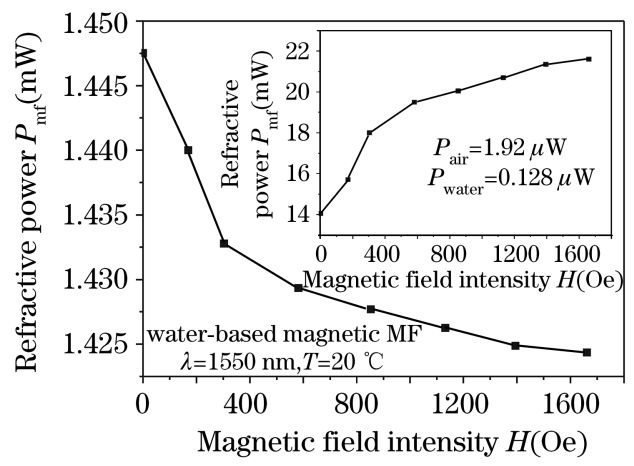

Fig. 2. Dependence of refractive indices of MF in bulk on the external magnetic field. electric field $\vec{E}$ and magnetic field $\vec{H}$. When $\vec{E}$ is perpendicular to $\vec{H}, \chi$ decreases with the increasing magnetic field, and when $\vec{E}$ is parallel to $\vec{H}, \chi$ increases with the increasing magnetic field. Since the refractive index $n=\sqrt{\varepsilon_{\mathrm{r}}}=\sqrt{1+\chi}$, the changing tendency of the refractive index of MF with the increasing external magnetic field will be dependent on the relative direction between the electric field $\vec{E}$ of the light source and the external magnetic field $\vec{H}$. In the experiments carried by Yang et al., the external magnetic field is perpendicular to the MF film and the relative direction between the magnetic field and the light in the MF film equals to the refractive angle of the refractive light from prism to the MF film, which can be found less than $45^{\circ}$. But in our experiment, the light in $\mathrm{MF}$ is the transmitted light from the detecting fiber to the $\mathrm{MF}$, and the relative direction between the magnetic field and the light in MF is around $90^{\circ}$ as shown in Fig. 1. Besides, Yang et al. did not report the polarization of the light source, which also determines the relative direction between the electric field $\vec{E}$ of the light in MF and the external magnetic field $\vec{H}$. Thus, in our experiment, the inevitable different relative directions between the electric field $\vec{E}$ of the light in MF and the external magnetic field $\vec{H}$ from those in the experiments of Yang et al. may lead to the contradiction between our results and the results by Yang et al..

In conclusion, the magnetic field-dependent refractive index of MF in bulk is measured with an optical alignment-free and highly accurate method. While the magnetic intensity increases from 0 to 1661 Oe, the refractive index of MF in bulk decreases from 1.4475 to 1.4244 , with a tuning range of refractive index of 0.0231 . The results lay out potential application of $\mathrm{MF}$ in tunable optical devices.

This work was supported by the National Natural Science Foundation of China (No. 10574092, 60377013), the "863" Project of China (2006AA01Z242), the "973" Project of China (2006CB806000), the Excellent Young Scholar (Dawn) Program by Shanghai Municipal Education Commission and the Program for New Century Excellent Talents in University of China. X. Chen is the author to whom the correspondence should be addressed, his e-mail address is xfchen@sjtu.edu.cn.

\section{References}

1. R. E. Rosensweig, Ferrohydrodynamics (Cambridge University Press, Cambridge, 1985).

2. P. A. Martinet, Rheol. Acta 13, 260 (1974).

3. S. Taketomi, M. Ukita, M. Mizukami, H. Miyajima, and S. Chikazumi, J. Phys. Soc. Jpn. 56, 3362 (1987).

4. N. A. Yusuf, H. Abu-Safia, and I. Abu-Aljarayesh, J. Appl. Phys. 73, 6136 (1993).

5. H. E. Horng, C.-Y. Hong, H. C. Yang, I. J. Jang, S. Y. Yang, J. M. Wu, S. L. Lee, and F. C. Kuo, J. Magn. Magn. Mater. 201, 215 (1999).

6. M. T. A. Elói, R. B. Azevedo, E. C. D. Lima, A. C. M. Pimenta, and P. C. Morais, J. Magn. Magn. Mater. 289, 168 (2005).

7. Z. Di, X. Chen, S. Pu, X. Hu, and Y. Xia, J. Appl. Phys. 89, 211106 (2006).

8. H.-E. Horng, C.-Y. Hong, W. B. Yeung, and H.-C. Yang, 
Appl. Opt. 37, 2674 (1998).

9. H.-E. Horng, S. Y. Yang, S. L. Lee, C.-Y. Hong, and H. C. Yang, Appl. Phys. Lett. 79, 350 (2001).

10. S. Pu, X. Chen, Y. Chen, Y. Xu, W. Liao, L. Chen, and Y. Xia, J. Appl. Phys. 99, 093516 (2006).

11. S. Pu, X. Chen, Y. Chen, W. Liao, Y. Chen, and Y. Xia, Appl. Phys. Lett. 86, 171904 (2005).

12. W. Luo, T. Du, and J. Huang, J. Magn. Magn. Mater. 201, 88 (1999).

13. W. Luo, T. Du, and J. Huang, Phys. Rev. Lett. 82, 4134 (1999).

14. S. Y. Yang, Y. F. Chen, H. E. Horng, C. Y. Hong, W. S. Tse, and H. C. Yang, Appl. Phys. Lett. 81, 4931 (2002).
15. S. Y. Yang, J. J. Chieh, H. E. Horng, Chin-Yih Hong, and H. C. Yang, Appl. Phys. Lett. 84, 5204 (2004).

16. C.-Y. Hong, H. E. Horng, and S. Y. Yang, Phys. Stat. Sol. (c) 1, 1604 (2004).

17. H. E. Horng, C. S. Chen, K. L. Fang, S. Y. Yang, J. J. Chieh, C. Y. Hong, and H. C. Yang, Appl. Phys. Lett. 85, 5592 (2004).

18. W. Liao, X. Chen, Y. Chen, S. Pu, Y. Xia, and Q. Li, Appl. Phys. Lett. 87, 151122 (2005).

19. S. Pu, X. Chen, Y. Chen, W. Liao, L. Chen, and Y. Xia, Appl. Phys. Lett. 86, 171904 (2005).

20. A. J. Mailfert and B. Nahounou, IEEE Trans. Magnet. MAG-16, 254 (1980). 